\title{
DE QUELQUES GRANDES QUESTIONS HUMANITAIRES TRAITÉES PAR LA XXV' CONFÉRENCE INTERNATIONALE DE LA CROIX-ROUGE
}

On l'a dit, la $X X V^{e}$ Conférence internationale de la Croix-Rouge (octobre 1986), dont la Revue internationale de la Croix-Rouge a largement relaté les travaux dans son numéro spécial de novembredécembre 1986, aura été marquée par la suspension de la participation de la délégation gouvernementale sud-africaine à la Conférence.

Cet événement ne doit cependant pas occulter les nombreux acquis de la Conférence qui sont autant l'expression de la dynamique de la Croix-Rouge internationale que celle de la volonté de ses membres de poursuivre et développer ses tâches humanitaires.

Deux grands thèmes ont dominé les travaux de la XXVe Conférence internationale: les nouveaux Statuts du "Mouvement international de la Croix-Rouge et du Croissant-Rouge» et le respect du droit international humanitaire.

Dans ce présent numéro la Revue publie le texte intégral des Statuts du Mouvement et de son Règlement, tels qu'ils ont été adoptés par la Conférence le 31 octobre 1986 (voir pp. 25-59). Un tiré à part en sera également fait.

Il est peu de dire que ces Statuts constituent une amélioration par rapport au texte de 1952. On peut, entre autres, souligner l'importance du Préambule qui, pour la première fois dans l'histoire législative du Mouvement, regroupe ce qui en fait la raison d'être et sa finalité et en anime l'action: sa mission fondamentale, ses Principes fondamentaux, ses devises et sa contribution à la paix.

La nouvelle appellation "Mouvement international de la CroixRouge et du Croissant-Rouge", également connu sous le nom de "Croix-Rouge internationale", souligne le caractère dynamique d'une 
institution qui, depuis sa création, vit pour et par l'action humanitaire universelle. De même ces Statuts ont le mérite de préciser le rôle de chacune des composantes du Mouvement, notamment celui des Sociétés nationales, et de mettre en relief la coopération des Etats parties aux Conventions de Genève avec les composantes du Mouvement. Leur adoption par consensus est un signe de bon augure.

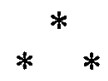

Le respect du droit international humanitaire constituait un des thèmes majeurs des travaux de la Conférence. Comme annoncé dans sa précédente livraison, la Revue publie dans ce numéro le texte intégral du rapport d'activité du CICR présenté le 27 octobre 1986 sous ce thème par le président Hay à la Commission du droit humanitaire de la Conférence (voir pp. 60-80).

Dans son précédent numéro, la Revue a rendu compte du débat animé que ce rapport a suscité au sein de la Conférence ${ }^{1}$. Rappelons ici que celle-ci a finalement adopté par consensus une résolution par laquelle elle exprime sa profonde préoccupation devant les difficultés opposées au CICR dans ses efforts pour protéger et assister toutes les victimes militaires et civiles des conflits armés. Elle fait appel à toutes les Parties engagées dans ces conflits pour qu'elles respectent pleinement leurs obligations, telles que prévues par le droit international humanitaire, et permettent au CICR d'exercer ses activités humanitaires.

La communauté des Etats n'est pas restée insensible à ces problèmes préoccupants. Preuve en est la résolution adoptée par l'Organisation des Etats Américains qui prie ses membres de continuer à appuyer la tâche du CICR (voir pp. 117-118) tandis que la dernière Assemblée générale des Nations Unies fait écho à la Conférence internationale de la Croix-Rouge lorsqu'à son tour, en novembre, elle demande aux Etats parties aux Conventions de Genève de 1949 de devenir aussi Parties aux Protocoles additionnels « $\dot{a}$ une date aussi rapprochée que possible» (voir pp. 118-120).

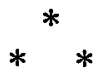

${ }^{\prime} R I C R, \mathrm{n}^{\circ} 762$, novembre-décembre 1986, pp. 339-341. 
Autres thèmes importants traités par la $X X V^{e}$ Conférence internationale: la protection des prisonniers de guerre et des civils pendant les conflits armés, la recherche et la réunion des familles. La Revue a le plaisir de publier à ce propos une étude d'un juriste du CICR sur les Bureaux nationaux de renseignements dont la tâche essentielle est d'obtenir et transmettre tous les renseignements disponibles sur les personnes protégées. L'auteur s'attache à en décrire et analyser les aspects juridiques, conventionnels, paraconventionnels et extraconventionnels ainsi que la pratique des Etats en la matière (voir pp. 6-24).

La Conférence internationale a également adopté d'importantes résolutions sur les Protocoles additionnels aux Conventions de Genève, la diffusion du droit international humanitaire et des principes et idéaux du Mouvement et les réfugiés. La Revue aura l'occasion, dans ses prochains numéros de l'année 1987, d'analyser, commenter et illustrer ces différents thèmes.

Mais en même temps la Revue désire s'ouvrir davantage au Mouvement qui vient de s'enrichir de nouvelles Sociétés nationales (voir pp. 81-90) et se faire l'écho des réalisations, des recherches et des préoccupations de ses composantes et notamment des Sociétés nationales de la Croix-Rouge et du Croissant-Rouge. L'action de la Croix-Rouge espagnole en faveur des marginaux en est un premier exemple (voir pp. 103-106) qui, nous l'espérons, sera suivi de nombreux autres.

Elle s'attachera enfin à informer ses lecteurs sur les grandes options humanitaires que partagent d'autres institutions humanitaires.

En présentant à ses fidèles lecteurs ses meilleurs væux pour 1987, la Revue, pour sa part, s'efforcera avec leur concours d'enrichir le patrimoine culturel du Mouvement tout en renforçant les liens entre ses membres.

La Revue 